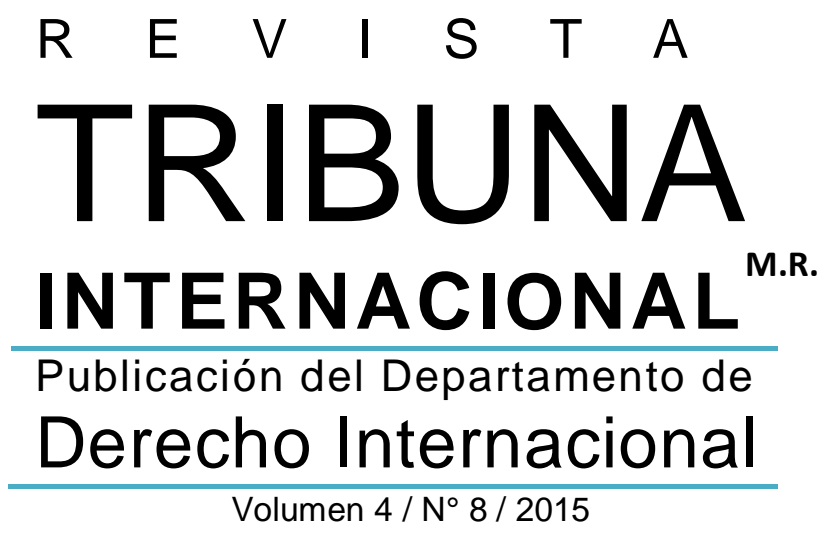

FACULTAD DE

DERECHO

UNIVERSIDAD DE CHILE 


\section{Rector de la Universidad de Chile}

Ennio Vivaldi Véjar

Av. Alameda Libertador Bernardo O'Higgins

1058, Santiago

\section{Representante legal}

Davor Harasic Yaksic

Decano de la Facultad de Derecho

Universidad de Chile

\section{Director Departamento Derecho}

Internacional

Edmundo Vargas Carreño

\section{Director (S) Revista Tribuna Internacional}

Mario Arnello Romo

\section{Editor General Revista Tribuna Internacional}

Luis Valentín Ferrada Walker

\section{Comité Editorial}

Íñigo Álvarez Gálvez (Universidad de Chile, Chile)

Gonzalo Aguilar (Universidad de Talca, Cbile)

José Carlos Fernández Rosas (Universidad

Complutense de Madrid, España)

Claudio Grossman (American University, EE.UU.)

Mattias Kumm (New York University, EE.UU.)

Hugo Llanos (Universidad Central, Chile)

Cecilia Medina (Universidad Diego Portales, Chile)

Elina Mereminskaya (Universidad de Chile, Chile)

Mónica Pinto (Universidad de Buenos Aires, Argentina)

\section{Fundador de la Revista Tribuna Internacional}

Mario Ramírez Necochea $†$

\section{Revista Tribuna Internacional M.R.}

Publicación del Departamento de Derecho Internacional de la Facultad de Derecho de la Universidad de Chile. Su objetivo es fomentar la reflexión, el debate, el análisis y la comunicación sobre el derecho internacional en forma pluralista y con rigor científico. Se publica cada semestre en los meses de junio y diciembre mediante convocatoria abierta a la publicación de artículos y monografías inéditos, comentarios de jurisprudencia, recensiones y comentarios de libros, en los campos de derecho internacional público y privado, derecho internacional de los derechos humanos y relaciones internacionales, tanto en castellano como inglés.

La Revista Tribuna Internacional fue creada por Decreto Exento No 8.466 de la Rectoría de la Universidad de Chile, de 22 de marzo del 2011.

Volumen 4/ No 8 / 2015

www.tribunainternacional.uchile.cl ISSN 0719-482X (versión en línea)

Departamento de Derecho Internacional Facultad de Derecho Universidad de Chile Av. Santa María 076, $4^{\circ}$ piso

Providencia, Santiago de Chile

\section{Diseño y producción:}

Facultad de Derecho

Universidad de Chile

Se autoriza la reproducción total o parcial del contenido de la publicación, siempre que se reconozca y cite el/ la/ los/ las autor/a/es/as y la publicación, no se realicen modificaciones a la obra y no se la utilice para fines comerciales. 


\title{
Protección en el trabajo para la comunidad LGBT ¿Qué ha dicho el Sistema Internacional? ${ }^{1}$
}

\author{
Workplace Protection for LGBT. What Is Been Said by the International System?
}

\begin{abstract}
Alejandro Cáceres Monroy
acaceresmonroy@gmail.com

Abogado y politólogo de la Universidad de los Andes (Bogotá, Colombia), con Maestría en Derecho Internacional y Comparado de la Universidad de California, Los Ángeles (UCLA) y estudios de género y sexualidad en la Universidad de Miami. Actualmente es Asesor en materias de género en el Ministerio del Trabajo de Colombia.
\end{abstract}

Resumen: Las personas lesbianas, gays, bisexuales o transgénero (LGBT) luchan actualmente por el reconocimiento de derechos civiles y políticos en muchos lugares del mundo. Sin ese piso de protección legal, pensar en derechos económicos, sociales y culturales es en muchas ocasiones una utopía. Bajo esta realidad, el lugar de trabajo se ha convertido en uno de los espacios donde más discriminación y dificultades para el goce de derechos sufre la comunidad LGBT.

El sistema de protección de derechos humanos se nutre principalmente de lo desarrollado por los tratados de derechos humanos, los comités de monitoreo y las agencias especializadas. Actualmente no existe un tratado para proteger los derechos de las personas LGBT, sin embargo avances interpretativos y desarrollos no vinculantes construidos por los Estados, han incentivado una nueva protección a los derechos más allá del contenido literal del texto normativo, lo que supone un avance para el derecho al trabajo de los trabajadores LGBT.

Palabras clave: Discriminación en el trabajo - orientación sexual - identidad de género Derecho Internacional.

\begin{abstract}
The lesbian, gay, bisexual and transgender people (LGBT) is currently fighting for the recognition of civil and political rights in many parts of the world. Without that legal protection, thinking on economic, social and cultural rights is often a utopia. Under this situation, the workplace has become one of the places where most discrimination occurs and difficulties in the enjoyment of rights are widely experienced by the LGBT community.

Human rights treaties, monitoring committees and specialized agencies, develop the system of buman rights protection. Currently there is no treaty to protect the rights of LGBT people; however interpretative advances and
\end{abstract}

1 Artículo enviado el 01.07.2015 y aceptado el 09.12.2015. 
Protección en el trabajo para la comunidad LGBT ¿Qué ha dicho el Sistema Internacional?

Alejandro Cáceres Monroy

non-binding developments built by the States have encouraged new rights protection beyond the literal content of the regulatory text that represents a progress for the right to work for LGBT workers.

Keywords: Workplace discrimination - sexual orientation - gender identity - International Law.

\section{Introducción}

Alrededor del mundo las personas LGBT han sido discriminadas en distintos escenarios. El hecho de que hoy por hoy todavía se debata sobre la igualdad formal en términos de derechos civiles y políticos da cuenta del largo camino que los movimientos LGBT y sus aliados han atravesado. El trabajo y los derechos y deberes asociados a la prestación personal de servicios, su remuneración y el estar sujeto a subordinación no han estado exentos de los problemas de discriminación. Hoy en día, en países donde se ha alcanzado igualdad formal respecto derechos civiles y políticos, los debates se movilizan alrededor de la eliminación y sanción de la discriminación en el empleo, vivienda y educación.

Trabajadores calificados son constantemente rechazados en oportunidades de trabajo, despedidos o discriminados simplemente porque son lesbianas, gays, bisexuales o transgénero. La situación de discriminación en el empleo de población LGBT no es fácil de cuantificar, pues aún existen sistemas precarios de recolección de información y las preguntas sobre orientación sexual e identidad de género brillan por su ausencia. Algunos centros de pensamiento han logrado cuantificar el impacto y la perdida social que ha significado esta discriminación, así el Williams Institute de la Universidad de California en Los Ángeles, identificó que en sólo en Estados Unidos "entre el 15\% y el 43\% de los trabajadores gays, lesbianas, bisexuales o transgénero han experimentado despedidos injustificados, se les han negado promociones o han sido acosados" ${ }^{\prime 2}$ Lo anterior se evidencia en el hecho de que casi dos de cada tres lesbianas o gays tienen que soportan bromas y comentarios negativos sobre su orientación sexual ${ }^{3}$, cerca del $31 \%$ de los empleados que han reconocido públicamente su condición temen perder la relación con sus compañeros ${ }^{4}$ y el $23 \%$ temen que no se les ofrezca desarrollo profesional u oportunidades de promoción ${ }^{5}$. Todo lo anterior contrasta con el

2 Vid. SEARS, Brad et al. "Documenting Discrimination on the Basis of Sexual Orientation and Gender Identity in State Employment”, 2009 [en línea] <http://williamsinstitute.law.ucla.edu/research/workplace/documenting-discriminationon-the-basis-of-sexual-orientation-and-gender-identity-in-state-employment/> [consulta: 17.dic.2015].

3 FIDAS, Deena y COOPER, Liz. "The Cost of the Closet and the Rewards of Inclusion: Why the Workplace Environment for LGBT People Matters to Employees”, en Human Rights Campaign, p. 16, 2015 [en línea] < http:/ /hrc-assets.s3-websiteus-east-1.amazonaws.com// files/assets/resources/Cost_of_the_Closet_May2014.pdf > [consulta: 17.dic.2015].

4 FIDAS y COOPER, "The Cost of the Closet...", op.cit., p. 16.

5 Fidas y COOPER, “The Cost of the Closet...”, op.cit., p. 16. 
Revista Tribuna Internacional

Volumen $4 \cdot \mathrm{N}^{\circ} 8 \cdot 2015 \cdot$ pp. 107-122

ISSN 0719-482X (versión en línea)

hecho de que solamente 61 países cuentan con legislación que proteja contra la discriminación en el empleo ${ }^{6}$.

Este texto explorará de qué manera el sistema jurídico internacional ha abordado la discriminación por motivos de orientación sexual e identidad de género, y su protección contra la discriminación en el lugar de trabajo. Primero se explorará en los textos de los tratados internacionales, decisiones de sus órganos de monitoreo y en la producción de soft law sobre provisiones generales antidiscriminación. Segundo, se estudiará el papel de la Organización Internacional del Trabajo y del Comité de Derechos Económicos, Culturales y Sociales respecto la protección en el empleo para la comunidad LGBT. Por último, desarrollará una conclusión que aboga por la producción de una política inclusiva y protectora, que reconozca las realidades y necesidades de la comunidad LGBT y promueva el entendimiento y desarrollo de normas internacionales incluyentes y protectoras sin necesidad de ocurrencias interpretativas.

\section{Derecho Internacional de los Derechos Humanos: Protección contra la discriminación a la comunidad LGBT}

En el sistema internacional de los derechos humanos no existe un tratado especial sobre la comunidad LGBT. Sin embargo, algunos de los órganos de monitoreo de los tratados (comités), han interpretado el contenido de algunos tratados generales de derechos humanos y sus disposiciones contra la discriminación, para incluir protección a la orientación sexual e identidad de género. Los tratados internacionales de derechos humanos fundamentales son el Pacto Internacional de Derechos Civiles y Políticos (PIDCP) y el Pacto Internacional de Derechos Económicos, Sociales y Culturales (PIDESC). Estos, junto con la Declaración Universal de los Derechos Humanos, constituyen la Carta Internacional de Derechos Humanos.

Para el propósito de este texto debe considerarse, siguiendo a Malcolm Shaw, que "ciertos derechos humanos ahora pueden ser considerados como parte de la categoría de derecho internacional consuetudinario a la luz de la práctica estatal ...incluyendo la prohibición de tortura, el genocidio, la esclavitud y el principio de no discriminación..."”. Así, estos derechos desarrollados en virtud de los tratados "pueden constituirse en obligaciones erga omnes para los Estados Partes. Sin embargo, algunas categorías que hacen parte del repertorio de la

6 Itaborahy, Lucas y ZhU, Jingshu. "State-sponsored Homophobia: World Survey of Laws: Criminalisation, Protection and Recognition of Same-sex Love", IGLA, 2014, p. 22 [en línea]

<http://old.ilga.org/Statehomophobia/ILGA_SSHR_2014_Eng.pdf> [consulta: 17.dic.2015].

7 SHAw, Malcolm. International Law. Cambridge, Cambridge University Press, 2008, p. 275. 
discriminación siguen siendo tema de debate, dentro de los que se encuentran la identidad de género y la orientación sexual.

El PIDCP es un tratado adoptado por la Asamblea General de las Naciones Unidas en 1976. Compromete a sus partes a respetar los derechos civiles y políticos de las personas, incluido el derecho a la vida, las libertades de religión, de expresión y de reunión, los derechos electorales y los derechos al debido proceso y a un juicio justo. Este pacto tiene 168 Estados Partes. El cumplimiento del PIDCP es supervisado por el Comité de Derechos Humanos, que revisa los informes periódicos de los Estados sobre cómo se aplica el tratado.

Para entender el nivel de protección de la comunidad LGBT, es necesario describir algunos de sus artículos más relevantes. Así, el artículo 2 (1) del PIDCP establece que:

"Cada uno de los Estados Partes en el presente Pacto se compromete a respetar y a garantizar a todos los individuos que se encuentren en su territorio y estén sujetos a su jurisdicción los derechos reconocidos en el presente Pacto, sin distinción alguna de raza, color, sexo, idioma, religión, opinión política o de otra índole, origen nacional o social, posición económica, nacimiento o cualquier otra condición social'. (cursivas agregadas)

Las disposiciones "sexo" y "cualquier otra condición social" podrían interpretarse como una prohibición de la discriminación por motivos de orientación sexual e identidad de género. De la misma manera el artículo 26 del PIDCP, dicta que:

"Todas las personas son iguales ante la ley y tienen derecho sin discriminación a igual protección de la ley. A este respecto, la ley prohibirá toda discriminación y garantizará a todas las personas protección igual y efectiva contra cualquier discriminación por motivos de raza, color, sexo, idioma, religión, opiniones políticas o de cualquier índole, origen nacional o social, posición económica, nacimiento o cualquier otra condición social'. (cursivas agregadas)

Una vez más, "cualquier otra condición social" puede ser interpretada como una protección para ciertos status que para el momento de la redacción del tratado no fueron identificados, pero que no por eso no se encontraban protegidos. Para una mejor comprensión de estas disposiciones como una fuente de protección de la comunidad LGBT, son útiles las decisiones tomadas por el Comité de Derechos Humanos. Así, en 1992 el Comité tuvo la oportunidad de decidir un caso sobre una pareja homosexual en Tasmania, denominado Toonen c. Australia ${ }^{8}$ El Comité estableció que “el término 'sexo' incluye la protección contra la discriminación basada en la orientación sexual. Al considerar si el derecho de Nicolás Toonen a la privacidad (artículo 17.1) otorgado por el Pacto fue violado por una ley de Tasmania que penaliza las relaciones sexuales consentidas entre dos hombres adultos, el Comité determinó que, de conformidad con el artículo 2 (1) del Pacto, Tasmania debe aplicar las protecciones del artículo 17 sobre el

8 Comité de Derechos Humanos de las N.U., Toonen c. Australia, Comunicación No. 488/1992, U.N. Doc. CCPR/C/50/D/488/1992 (1994). 
Revista Tribuna Internacional

Volumen $4 \cdot \mathrm{N}^{\circ} 8 \cdot 2015 \cdot$ pp. 107-122

ISSN 0719-482X (versión en línea)

derecho a la intimidad del PIDCP, sin discriminación sobre la base de "sexo". Así pues "la referencia a 'sexo' en los artículos 2, y el apartado 1 del artículo 26 incluyen la orientación sexual" "10 por lo que el Comité resolvió que el Commonwealth de Australia violó los derechos del Sr. Toonen respecto el artículo 2 (1) ya que el Gobierno de Tasmania discriminó contra las personas homosexuales en la aplicación de los derechos reconocidos por el Pacto ${ }^{11}$.

El Comité también interpretó la frase "cualquier otra condición social" para prohibir la discriminación por los mismos motivos que Toonen, declarando que "la orientación sexual es una de las 'cualquier otra condición social' que puede existir para los efectos del [PIDCP y] (...) parece haber un fuerte argumento de que las palabras de los dos artículos no deben interpretarse de forma restrictiva" ". La formulación de tales disposiciones, "sin distinción de ningún tipo" y "cualquier otro índole", admiten una interpretación inclusiva y no una exhaustiva.

Diez años después, en el caso Young c. Australia, donde la negativa del Estado Parte a conceder al demandante una pensión sobre la base de que no cumplía con la definición de "dependiente", por haber estado en una relación del mismo sexo con el Sr. C, vulnera sus derechos en virtud del artículo 26 del Pacto, sobre la base de su orientación sexual, el Comité recuerda:

“...su jurisprudencia anterior sobre la prohibición de la discriminación en virtud del artículo 26 que comprende también la discriminación basada en la orientación sexual. [...] [El Comité insiste en que] no toda distinción constituye una discriminación prohibida por el Pacto, siempre que esta se base en criterios razonables y objetivos. El Estado Parte no proporciona argumentos sobre cómo esta distinción entre parejas del mismo sexo, que están excluidos de los beneficios de pensión en virtud de la ley, y las parejas heterosexuales no casadas, a quienes sí se le otorgan estos beneficios, es razonable y objetiva, y no hay pruebas que apuntan a la existencia de factores que justifiquen tal distinción. En este contexto, el Comité considera que el Estado Parte ha violado el artículo 26 del Pacto al denegar al peticionario una pensión sobre la base de su sexo u orientación sexual"'13.

En otro caso, $X$ c. Colombia, sobre una transferencia de pensiones, el peticionario, presentó una solicitud ante el Fondo de Bienestar Social para una transferencia de pensiones, la petición fue denegada por el Fondo y ningún recurso legal fue exitoso. Una vez más, el Comité insiste en la idea de que la prohibición de la discriminación en virtud del artículo 26 comprende también la discriminación basada en la orientación sexual y encuentra que:

Comité de Derechos Humanos de las N.U., Toonen c. Australia..., op.cit., \8.7.

10 Comité de Derechos Humanos De Las N.U., Toonen c. Australia..., op.cit., $\int 8.7$.

11 Comité de Derechos Humanos de las N.U., Toonen c. Australia..., op.cit., \11.

12 Comité de Derechos Humanos De las N.U., Toonen c. Australia..., op.cit., SS 6.9 y 8.7.

13 Comité de Derechos Humanos de las N.U., Young c. Australia, Comunicación No. 941/2000, U.N. Doc CCPR/C/78/D/941/2000 (2003). 
"el Estado Parte no ha aportado ningún argumento que pueda demostrar que la distinción entre parejas del mismo sexo, que no tienen derecho a las prestaciones de jubilación, y las parejas heterosexuales no casadas, que si tienen el derecho, es razonable y objetiva. [...] El Comité considera que el Estado Parte ha violado el artículo 26 del Pacto al negar el derecho del peticionario a la pensión de su compañero de vida sobre la base de su orientación sexual"14.

Esos son todos los casos sobre la orientación sexual presentados hasta la fecha ante el Comité de Derechos Humanos y está bien establecido que a pesar de que no existe ninguna disposición especial para la población $\mathrm{LGBT}^{15}$, la interpretación del Pacto establece la protección de la discriminación por motivos de orientación sexual bajo la cláusula de "sexo" y "otra condición social".

Además de las decisiones de casos por parte del Comité, otra de las responsabilidades de los Estados en virtud del Pacto es presentar Informes Periódicos sobre el cumplimiento de sus obligaciones. Estos informes se enfrentan a informes sombra de las ONG, determinándose las observaciones finales formuladas por el Comité al Estado Parte. Ellas son evaluaciones de la aplicación de los tratados de derechos humanos por parte del Estado. También contienen recomendaciones para mejorar la aplicación de los derechos del mismo. Las observaciones finales no imponen ninguna obligación legal a los Estados Partes. En cualquier caso, el texto de la mayoría de las observaciones finales aprobadas hasta el momento deja claro que los órganos de los tratados ven su papel como de asesoría y recomendación ${ }^{16}$.

Así, en las Observaciones Finales respecto a Chile del año 2007, el Comité “observa con satisfacción que las leyes que penalizan las relaciones homosexuales consentidas entre adultos han sido derogadas", aunque observó que "el Estado Parte debe garantizar la igualdad de derechos para todas las personas, conforme a lo establecido en el [PIDCP], independientemente de su orientación sexual, incluyendo la igualdad ante la ley y el acceso a la atención en salud" ${ }^{\prime 17}$.

Como señalan Michael O'Flaherty y John Fisher el Comité "con frecuencia se plantea la cuestión de la discriminación por motivos de orientación sexual: durante el período 2000-06, lo hizo con respecto a 13 de los 84 países analizados. Criticó la criminalización de las relaciones sexuales homosexuales (varios países) ${ }^{18}$, el fracaso para prohibir la discriminación relacionada

14 Comité de Derechos Humanos de las N.U, X c. Colombia, Comunicación No. 1361/2005, U.N. Doc CCPR/C/89/D/1361/2005 (2007).

15 Todos los casos han sido de orientación sexual y no de orientación de género y aunque uno podría utilizar los mismo argumentos sobre interpretación extensiva y no restrictiva de las clausulas sexo y otra condición, aún no se conocen casos por discriminación a población transexual en el Comité de Derechos Humanos de las N.U..

16 Vid. O’FlaherTy, Michael. “The Concluding Observations of United Nations Human Rights Treaty Bodies”, en Human Rights Law Review, vol. 6, No 1, 2006, [en línea] <http://hrlr.oxfordjournals.org/content/6/1/27.full.pdf> [consulta: 17.dic.2015].

17 Comité de Derechos Humanos de las N.U., Obervaciones Finales a Chile, 17 de abril del 2007, U.N. Doc. $\mathrm{CCPR} / \mathrm{C} / \mathrm{CHL} / \mathrm{CO} / 5, \$ 16$.

18 Vid. Comité De Derechos Humanos De Las N.U., Obervaciones Finales a Egipto, 28 de noviembre del 2002, CCPR/CO/76/EGY, \$19; y Observaciones Finales a Kenia, CCPR/CO/83/KEN, 29 de abril del 2005, § 27. 
con el empleo, la no inclusión de la categoría de orientación sexual en las leyes antidiscriminación (varios países) ${ }^{19}$, la falta de programas de educación para luchar contra las actitudes discriminatorias y las edades desiguales de consentimiento para la actividad sexual $^{20,21}$.

Por otro lado, el PIDESC es un tratado adoptado por la Asamblea General de las Naciones Unidas, en vigor desde 1976. Este tiene por objeto garantizar la protección de los derechos económicos, sociales y culturales, incluido el derecho a la libre determinación de todos los pueblos, la igualdad de derechos entre hombres y mujeres, y el derecho al trabajo, entre otros. El PIDESC tiene 162 Partes. El Comité de las Naciones Unidas sobre Derechos Económicos, Sociales y Culturales (Comité DESC) supervisa el cumplimiento del Pacto. Para entender el nivel de protección de la comunidad LGBT en virtud de este tratado, es necesario tener en cuenta su artículo 2 (2), que establece que:

"Los Estados Partes en el presente Pacto se comprometen a garantizar el ejercicio de los derechos que en él se enuncian, sin discriminación alguna por motivos de raza, color, sexo, idioma, religión, opinión política o de otra índole, origen nacional o social, posición económica, nacimiento o cualquier otra condición social' (cursivas agregadas).

Las disposiciones resaltadas en el presente artículo marcan una idea de cómo se produce la interpretación del Pacto. No obstante, este tratado es más específico acerca de los derechos que el PIDCP, el PIDESC está escrito en positivo, y las obligaciones del Estado no son para que se abstengan de poner en peligro el disfrute del derecho, sino para cumplir el desarrollo de políticas para llevarlos a cabo.

En 2009 el Comité DESC publicó la Observación General No. 20 sobre "la no discriminación en los derechos económicos, sociales y culturales"22 en respeto con el artículo 2 (2) del Pacto, estableciendo que:

19 Vid. Comité de Derechos Humanos De las N.U., Obervaciones Finales a Trinidady Tobago, 3 de noviembre del 2000, CCPR/CO/70/TTO, \11; Observaciones Finales a El Salvador, 22 de julio del 2003, CCPR/CO/78/SLV, \16; Observaciones Finales a Filipinas, 01 de diciembre del 2003, CCPR/CO/79/PHL, \18; Observaciones Finales a Namibia, 30 de julio del 2004, CCPR/CO/81/NAM, \22; y Observaciones Finales a Polonia, 2 de diciembre del 2004, CCPR/CO/82/POL, $\int 18$.

20 Comité de Derechos Humanos de las N.U., Obervaciones Finales a Austria, 19 de noviembre de 1998, CCPR/C/79/Add.103, \13.

21 O'FlAHERTY, Michael y FiSHer, Jhon. "Sexual Orientation, Gender Identity and International Human Rights Law: Contextualising [sic] the Yogyakarta Principles", en Human Rights Law Review, vol. 8, No 2, 2008, [en línea] $<$ https://globalfop.files.wordpress.com/2012/11/sexual-orientation-gender-identity-and-international-human-rights-lawcontextualising-the-yogyakarta-principles.pdf $>$ [consulta: 17.dic.2015].

22 Los Comentarios Generales o Recomendaciones Generales (CG/RG) son parte de la tarea de supervisión de los Comités de Derechos Humanos y DESC. En ellos se tratan cuestiones de carácter general, abordando a todos los Estados Partes en lugar de un Estado Parte en particular, son interpretaciones autorizadas de los derechos humanos individuales o de la naturaleza jurídica de las obligaciones de derechos humanos, proporcionan orientaciones para la aplicación práctica de los derechos humanos y forman un conjunto de criterios para evaluar el progreso de los Estados en la implementación de derechos. Estrictamente hablando los CG/RG no son jurídicamente vinculantes, pero tienen un carácter altamente autorizado con una base legal sólida. Vid. Nisuke, Ando. General Comments/Recommendations, Max Planck Institute For Comparative Public Law And International Law, 2010, [en línea] 
“7. La no discriminación es una obligación inmediata y transversal en el Pacto. El Artículo 2 (2) exige a los Estados Partes garantizar la no discriminación en el ejercicio de cada uno de los derechos económicos, sociales y culturales consagrados en el Pacto y sólo puede aplicarse en conjunción con estos derechos. Es de señalar que la discriminación constituye toda distinción, exclusión, restricción o preferencia u otro trato diferenciado que se basa directa o indirectamente de los motivos probibidos de discriminación y que tengan por objeto o por resultado anular o menoscabar el reconocimiento, goce o ejercicio, en pie de igualdad, de los derechos reconocidos en el Pacto. La discriminación también incluye la incitación a la discriminación y el acoso". (cursivas agregadas)

Esta forma amplia de entender la discriminación es un logro increíble en el ámbito internacional, y establece unos estándares muy altos a los Estados Partes para no estar en violación del Pacto, también incluye el efecto de la discriminación y no sólo la intención, lo que en términos probatorios es un avance importante.

La Recomendación General No. 20 también establece que:

"27. La naturaleza de la discriminación varía según el contexto y evoluciona con el tiempo. Un enfoque flexible para el piso de 'cualquier otra condición' es necesario para capturar otras formas de trato diferenciado que no pueden ser razonable y objetivamente justificados y de naturaleza comparable a los motivos reconocidos expresamente en el artículo 2(2). Estos motivos adicionales son comúnmente reconocidos cuando reflejan la experiencia de los grupos sociales que son vulnerables y que ban sufrido y siguen sufriendo marginación. [...]

32. Orientación sexual e identidad de género 'cualquier otra condición' como se reconoce en el articulo 2 (2) incluye la orientación sexual. Los Estados Partes deben garantizar que la orientación sexual de una persona no es un obstáculo para la realización de los derechos reconocidos en el Pacto, por ejemplo, en el acceso a la pensión de viudez. Además, la identidad de género se reconoce como uno de los motivos prohibidos de discriminación; por ejemplo, las personas que son transgénero, transexuales o intersexuales a menudo enfrentan graves violaciones de los derechos humanos, como el acoso en las escuelas o en el lugar de trabajo". (cursivas agregadas)

Estos dos párrafos ejemplifican una comprensión completa de la discriminación que sufren las personas LGBT, no sólo por nombrar a la comunidad de manera directa, sino porque trae un nivel estricto de control de los Estados para cumplir con el articulado del Pacto. Acá no sólo la orientación sexual se incluye en la categoría de "cualquier otra condición social", sino que no deja ninguna duda de que la aplicación de la cláusula de no discriminación del Pacto incluye la identidad de género.

Además de los tratados, desde 2001 han habido varios intentos de producir material no vinculante (soft law) para la protección y cumplimiento de los derechos de las personas LGBT.

<http://ilmc.univie.ac.at/uploads/media/general_comments_recommendations_empil.pdf> [consulta: 17.dic.2015]. 
Así, por ejemplo, Brasil presentó el 2003 una propuesta de Resolución General relativa al reconocimiento de los derechos humanos de las personas de diversa orientación sexual e identidades de género, aunque luego fue retirada debido a ciertas presiones. Ha habido también diferentes declaraciones patrocinadas y presentadas por países europeos y latinoamericanos en los distintos foros de las Naciones Unidas, tales como la denominada "Terminando con los actos de violencia y violaciones relacionadas derechos humanos basadas en la orientación sexual y la identidad de género", redactada en marzo de 2011 y entregado por Colombia a la $16^{a}$ sesión del Consejo de Derechos Humanos de las Naciones Unidas en nombre de 85 Estados. Finalmente, hay una resolución de dicho Consejo, aprobada el 26 de septiembre de 2014, y destinada a combatir la violencia y la discriminación basada en la orientación sexual y la identidad de género, que estableció que "Los Estados deben revisar y derogar las leyes discriminatorias y abordar la discriminación contra las personas LGBT y personas intersexuales, incluidos el goce de los derechos a la salud, educación, trabajo, agua, vivienda adecuada y la seguridad social" ${ }^{23}$.

Estas iniciativas han sido celebradas por activistas, abogados, académicos y la comunidad LGBT. El 2006 un grupo de expertos sobre derechos humanos y activistas de la comunidad LGBT en todo el mundo desarrollaron una serie de principios llamados los Principios de Yogyakarta, relativos al "derecho internacional de los derechos humanos que se aplica a personas de diversas identidades de orientación sexual y de género, que establecen las obligaciones de los Estados para hacer que las personas LGBTI pueden disfrutar de sus derechos en las mismas condiciones que todos los demás en la sociedad"24.

Por último, el Tribunal Europeo de Derechos Humanos y la Comisión y la Corte Interamericana en menor medida, han sido prolíficos en la creación y el mantenimiento del sistema de protección de la comunidad LGBT. Sin embargo, un resumen de sus logros va más allá del alcance y el propósito de este trabajo ${ }^{25}$.

23 Consejo de Derechos Humanos de la N.U., 29a sesión, ítems 2 y 8 de la agenda; Informe Anual del Alto Comisionado de las N.U. para los Derechos Humanos e informes de la Oficina del Alto Comisionado y del Secretario General,

"Discrimination and violence against individuals based on their sexual orientation and gender identity", publicado el 4 de mayo del 2015, hasta el momento sólo en inglés.

24 Onufer CorrêA, Sonia y MuntarbHorn, Vitit. “An activist Guide to The Yogyakarta Principles”, 2010, [en línea] <http://issuu.com/lgbtexcellencecentre/docs/activists_guide_english_nov_14_2010> [consulta: 17.dic.2015]. La letra "I" que en la cita se ha agregado al final de la sigla LGTB, se refiere a las personas de condición "intersexual".

25 Para una mirada más amplía del sistema Europeo de protección para las personas LGBT vid. O’FLAHERTY, y FISHER, "Sexual Orientation...", op.cit., p. 14; y las decisiones y jurisprudencia agrupadas por la Relatoría de la Comisión Interamericana de Derechos Humanos sobre los derechos de las personas LGBTI, [en línea]

<http://www.oas.org/es/cidh/lgtbi/decisiones/cidh.asp> [consulta 18.dic.2015]. 


\section{E1 derecho al trabajo para la comunidad LGBT en el Sistema Internacional}

La Organización Internacional del Trabajo (OIT) ha impulsado el desarrollo del concepto de trabajo digno y justo, las buenas condiciones de trabajo, la salud de los trabajadores, la lucha contra la esclavitud y la trata sobre las personas, entre otros temas relacionados con cuestiones laborales.

La OIT es un organismo de las Naciones Unidas y establece las normas internacionales del trabajo, tiene 185 Miembros que reúnen a representantes de los gobiernos, los empleadores y los trabajadores. La OIT registra quejas contra entidades que violan las normas internacionales; sin embargo, no impone sanciones a los gobiernos. En 1960, la OIT desarrolló el Convenio 111 sobre la discriminación en el empleo y la ocupación. Tal Convenio establecer que el término discriminación comprende:

“(a) cualquier distinción, exclusión o preferencia basada en motivos de raza, color, sexo, religión, opinión política, ascendencia nacional u origen social que tenga por efecto anular o alterar la igualdad de oportunidades o de trato en el empleo y la ocupación;

(b) cualquier otra distinción, exclusión o preferencia que tenga por efecto anular o alterar la igualdad de oportunidades o de trato en el empleo u ocupación que podrá ser especificada por el Miembro interesado previa consulta con las organizaciones representativas de empleadores y de trabajadores, cuando dichas organizaciones existan, y con otros organismos apropiados".

Esta definición no prohíbe expresamente la discriminación por motivos de orientación sexual, sin embargo permite a los Estados Partes añadir motivos adicionales. Por ejemplo, la aplicación de la Convención en el derecho interno en Australia contribuyó a la eliminación de la prohibición de mujeres lesbianas y hombres gay en las fuerzas armadas en 1992. Desde 1998, la $86^{\mathrm{a}}$ Conferencia Internacional del Trabajo adoptó la "Declaración sobre principios y derechos fundamentales en el trabajo", como una expresión de compromiso de los gobiernos, organizaciones de empleadores y de trabajadores para afianzar los valores humanos básicos, valores que son vitales para nuestra vida económica y social ${ }^{26}$. Esta declaración contiene cuatro políticas fundamentales: "El derecho de los trabajadores a asociarse libremente y negociar colectivamente; la eliminación del trabajo forzoso y obligatorio; la eliminación del trabajo infantil; y el fin de la discriminación injusta entre los trabajadores". La declaración señala que:

26 Vid. OIT, "Declaration on Fundamental Principles and Rights at Work", [en línea]

<http://www.ilo.org/declaration/lang--en/index.htm> [consulta 18.dic.2015]. 
Revista Tribuna Internacional

Volumen $4 \cdot \mathrm{N}^{\circ} 8 \cdot 2015 \cdot$ pp. 107-122

ISSN 0719-482X (versión en línea)

"Todos los Miembros, aun cuando no hayan ratificado los convenios aludidos [incluido el 111], tienen un compromiso que se deriva de su mera pertenencia a la Organización de respetar, promover y hacer realidad, de buena fe y de acuerdo con la Constitución de la OIT, los principios relativos a los derechos fundamentales que son objeto de esos convenios".

Es decir, las cuatro políticas fundamentales que se desarrollan respectivamente en convenios contienen obligaciones básicas que deben ser promovidas por los Estados Partes de la Organización con independencia de si son o no parte del convenio que la desarrolla.

En 1990, la Comisión de Expertos en Aplicación de Convenios y Recomendaciones (CEACR) llamó la atención por primera vez sobre disposiciones legislativas relativas a la discriminación basada en la orientación sexual, y destacó, en su "Estudio Especial de 1996 sobre la Igualdad en el Empleo y Ocupación”, que la orientación sexual era un motivo emergente de discriminación en el lugar de trabajo. La CEACR consideró que, con miras a garantizar una protección específica contra toda discriminación basada en la orientación sexual, es conveniente prever legislación nacional al respecto ${ }^{27}$. Uno de los mayores avances de la OIT fue consagrar desde el 2003 que la discriminación relacionada con la orientación sexual y la orientación de género se reportara en los informes globales ${ }^{28}$. Desde mayo del 2014, la OIT lleva adelante un proyecto para el desarrollo de la nueva norma en materia de orientación sexual e identidad de género en el lugar de trabajo ${ }^{29}$. Últimamente, la CEACR ha puesto atención en estas materias, y de hecho en su Informe Mundial (2007) hizo hincapié en las manifestaciones más comunes de discriminación en el lugar de trabajo:

"Denegación de empleo, despido, denegación de ascenso.

Acoso: bromas indeseables, indirectas y comentarios tendenciosos, abuso verbal, chismes difamatorios, apodos, intimidación y hostigamiento, falsas acusaciones de pedofilia, pintadas, llamadas telefónicas insultantes, anónimos, daños a los bienes, chantaje, violencia e incluso amenazas de muerte.

Denegación de prestaciones a la pareja del mismo sexo (por ejemplo, días de asueto adicionales por diferentes razones como traslado, nacimiento de un hijo, licencia parental, cuidado de la pareja enferma, pérdida de un familiar; prestaciones educacionales para los trabajadores y su familia; suministro de bienes y servicios de calidad por parte del empleador; prestaciones de supervivencia en los regímenes de

27 Vid. OIT, "Equality at Work: Tackling the Challenges", [en línea]

$<$ http://www.ilo.org/public/portugue/region/eurpro/lisbon/pdf/equality_07.pdf> [consulta 18.dic.2015].

28 OIT, "Equality at Work...", op.cit.

29 Identidad de Género y Orientación Sexual: Promoción de los derechos, la diversidad y la igualdad en el mundo del trabajo (PRIDE por sus siglas en inglés), es un proyecto de la OIT en que se investiga cómo la discriminación contra las personas LGBT se manifiesta en el lugar de trabajo. El estudio se utilizará como base para trabajar con los gobiernos y las organizaciones de trabajadores y de empleadores para promover los derechos, la diversidad y la tolerancia en el lugar de trabajo. Él se está desarrollando en Argentina, Sudáfrica, Hungría y Tailandia. Se esperan los resultados en el 2015, [en línea]

<http://www.ilo.org/gender/Projects/WCMS_184212/lang--es/index.htm> [consulta 18.dic.2015]. 
pensiones profesionales o a los efectos del seguro de vida, y seguro de enfermedad para los trabajadores y su familia).

Autoexclusión (por ejemplo, cuando los homosexuales evitan ciertos empleos, carreras o empleadores por temor a sufrir discriminación por su orientación sexual)" ${ }^{\prime 30}$.

A pesar de todas las anteriores iniciativas positivas y necesarias, la OIT, la agencia más importante en lo que respecta a los derechos laborales en todo el mundo, no tiene ninguna disposición específica que proteja a la comunidad LGBT, la CEACR ha sido restrictiva respecto la interpretación de la palabra "sexo" en la Convención 111, y ha decidido actuar de forma pasiva y sólo ha felicitado a los Estados que han desarrollado legislación contra la discriminación, pero no le ha pedido a los demás hacerlo. Los tímidos intentos para desarrollar estándares internacionales de protección de la discriminación por motivos de orientación sexual o identidad de género, no han dignificado las necesidades y la relevancia que la Organización tiene en el tema, su tardía decisión de desarrollar un estudio es sólo otra señal de la poca relevancia y centralidad que la Organización le da al tema.

Por otro lado, el PIDESC tiene una disposición respecto al derecho al trabajo, concretamente su artículo 6 (1), el que establece que:

“1. Los Estados Partes en el presente Pacto reconocen el derecho a trabajar, que comprende el derecho de toda persona a tener la oportunidad de ganarse la vida mediante un trabajo libremente escogido o aceptado, y tomarán medidas adecuadas para garantizar este derecho".

Mientras que el artículo 7, establece que los Estados Partes:

"Reconocen el derecho de toda persona al goce de condiciones de trabajo equitativas y satisfactorias que le aseguren en especial [...] c) Igual oportunidad para todos de ser promovidos, dentro de su trabajo, a la categoría superior que les corresponda, sin más consideraciones que los factores de tiempo de servicio y capacidad".

Estas disposiciones generales, como se ha visto en el apartado 2 de este texto, pueden interpretarse de forma restringida o amplia; el Comité DESC y su Observación General N ${ }^{\circ} 18$ iluminan sobre la forma en la que se entienden:

"1. El derecho al trabajo es un derecho fundamental, reconocido en diversos instrumentos de derecho internacional [...] El derecho al trabajo es esencial para la realización de otros derechos humanos y constituye una parte inseparable e inherente de la dignidad humana. Toda persona tiene el derecho a trabajar para poder vivir con dignidad. [...]

30 OIT, “Equality at Work...”, op.cit. 
Revista Tribuna Internacional

Volumen $4 \cdot \mathrm{N}^{\circ} 8 \cdot 2015 \cdot$ pp. 107-122

ISSN 0719-482X (versión en línea)

4. El derecho al trabajo, amparado en el PIDESC, afirma la obligación de los Estados Partes de garantizar a las personas su derecho al trabajo libremente elegido o aceptado, en particular el derecho a no ser privado de trabajo de forma injusta. Esta definición subraya el hecho de que el respeto a la persona y su dignidad se expresa a través de la libertad del individuo para elegir un trabajo, haciendo hincapié al tiempo en la importancia del trabajo para el desarrollo personal, así como para la integración social y económica. [...]

6. [...] El derecho al trabajo no debe entenderse como un derecho absoluto e incondicional a obtener empleo [...] Ello incluye el derecho de todo ser humano a decidir libremente aceptar o elegir trabajo. También supone no ser obligado de alguna manera a ejercer o efectuar un trabajo y el derecho de acceso a un sistema de protección que garantice a cada trabajador su acceso a empleo. Además implica el derecho a no ser privado injustamente del empleo. [...]

12. El ejercicio laboral en todas sus formas y a todos los niveles supone la existencia de los siguientes elementos interdependientes y esenciales, cuya aplicación dependerá de las condiciones existentes en cada Estado Parte:

a) Disponibilidad. Los Estados Partes deben contar con servicios especializados que tengan por función ayudar y apoyar a los individuos para permitirles identificar el empleo disponible y acceder a él.

b) Accesibilidad. El mercado del trabajo debe poder ser accesible a toda persona que esté bajo la jurisdicción de los Estados Partes. La accesibilidad reviste tres dimensiones:

i) En virtud del párrafo 2 del artículo 2, así como del artículo 3, el Pacto proscribe toda discriminación en el acceso al empleo y en la conservación del mismo por motivos de raza, color, sexo, idioma, religión, opinión política o de otra índole, origen nacional o social, posición económica, nacimiento, discapacidad física o mental, estado de salud (incluso en caso de infección por el VIH/SIDA), orientación sexual, estado civil, político, social o de otra naturaleza, con la intención, o que tenga por efecto, oponerse al ejercicio del derecho al trabajo en pie de igualdad, o hacerlo imposible. Según el artículo 2 del Convenio No 111 de la OIT, los Estados Partes deben 'formular y llevar a cabo una politica nacional que promueva, por métodos adecuados a las condiciones y a la práctica nacionales, la igualdad de oportunidades y de trato en materia de empleo y ocupación, con objeto de eliminar cualquier discriminación a este respecto"'. (cursivas agregadas)

El Comité DESC amplía el concepto de discriminación con el fin de proteger a más personas; "estas nuevas obligaciones conllevan ambas obligaciones, la negativa de abstenerse de interferencia y la obligación positiva de actuar" ${ }^{21}$. Eso significa derribar disposiciones que prohíban a las personas LGBT participar en la fuerza de trabajo, tales como las prohibiciones

31 Coomans, Fons, "Education and Work", en International Human Rights Law, Oxford, Oxford University Press, 2010, pp. 280-303. 
de acceder a empleos militares o los requisitos de prestación del servicio militar para acceder a empleos en el Estado de personas transexuales. Al mismo tiempo, esto significa crear escenarios de protección contra la discriminación, tal como promulgar legislación contra la discriminación por orientación sexual e identidad de género en el lugar de trabajo. Y, finalmente, exige a los Estados elaborar políticas activas de empleo para combatir el desempleo de las personas LGBT.

Con este rasero el Comité se ha pronunciado en observaciones finales a países. Así, en las observaciones a la República Islámica de Irán se le recomendó:

"Combatir y prevenir la discriminación y el estigma social contra los miembros de la comunidad de lesbianas, gays, bisexuales y transgénero, y asegurar el goce de todos los derechos consagrados en el Pacto, como el acceso sin trabas al empleo, los servicios sociales, la atención de la salud y la educación". ${ }^{32}$ (cursivas agregadas)

En el mismo sentido, en las Observaciones Finales al Estado de Jamaica se recomienda:

"Enmendar sus leyes para adoptar una ley general marco contra la discriminación, de conformidad con el artículo 2, párrafo 2, del Pacto, y teniendo en cuenta la Observación General No 20 (2009) sobre la no discriminación en los derechos económicos, sociales y culturales.

Al Comité le preocupa que relaciones del mismo sexo consensuales siguen siendo criminalizadas bajo la Ley de Delitos contra la Persona, perpetrando de este modo la discriminación contra los homosexuales, bisexuales y transexuales en todas las esferas de la vida, incluyendo el disfrute de los derechos económicos, sociales y culturales (art. 2). $[\ldots]$

(b) Adoptar medidas concretas, deliberadas y específicas para eliminar la discriminación por motivos de orientación sexual; y (c) Enviar un mensaje público claro de que cualquier forma de discriminación, acoso o violencia contra personas por su orientación sexual no es tolerada, y rápida y efectivamente investigar, procesar y sancionar a las personas por tales actos"33. (cursivas agregadas)

De esta manera se evidencia una interpretación uniforme del Comité DESC respecto los artículos que prohíben la discriminación por motivos de orientación sexual e identidad de género. El fracaso de los Estados en garantizar el principio de no discriminación es visto como una violación de otros derechos reconocido en el Pacto, como es el derecho al trabajo.

32 Comité ECOSOC, Observaciones Finales sobre la República Islámica de Irán, 29 de abril del 2014, U.N. Doc. $\mathrm{EC} / 12 / \mathrm{IRN} / \mathrm{CO} / 2$.

33 Comité ECOSOC, Observaciones Finales sobre Jamaica, 10 de junio del 2013, U.N. Doc. E/C.12/JAM/CO/3-4, $₫ 8$-9. 
Por último, respecto al PIDCP, y aunque no tiene mayores provisiones sobre el derecho al trabajo ${ }^{34}$, el Comité de Derechos Humanos en las Observaciones Finales a los Estados Unidos de América, resaltó:

"[...] con preocupación el hecho de no prohibir la discriminación en el empleo por motivos de orientación sexual en muchos estados [del Estado Parte] debe reconocer su obligación jurídica en virtud de los artículos 2 y 26 de garantizar a todos los derechos reconocidos por él [PIDCP], así como la igualdad protección ante la ley, sin discriminación por motivos de orientación sexual”35.

Indudablemente, la fortaleza del sistema internacional de protección de los derechos humanos hacia la comunidad LGBT ha aumentado. Los pactos de las Naciones Unidas y sus comités tienen una interpretación bien establecida sobre las cláusulas antidiscriminación y su aplicación a la población LGBT bajo el término "sexo" o la cláusula de "cualquier otra condición social". No obstante, las disposiciones emanadas de los comités no son de obligatorias y apenas tiene autoridad interpretativa; sin perjuicio que sus opiniones han incentivado la promulgación de leyes nacionales y ayudando a los defensores locales en la concreción de una legislación más protectora.

Al mismo tiempo, el reconocimiento que los comités han hecho de los Principios de Yogyakarta ha ayudado a los defensores de estos derechos en la adquisición de una mayor y mejor legitimidad en la promoción de agendas LGBT en la legislación nacional e internacional. La Asamblea General de Naciones Unidas y algunos Estados miembros de la misma (la mayoría de ellos europeos y latinoamericanos) han persistido en situar en la agenda internacional las necesidades de protección contra la violencia y la discriminación de la comunidad LGBT. Esto sin duda ha ayudado a la formación de una mayor aceptación en la comunidad global, incluso con la oposición directa y frontal de algunos Estados (por ejemplo los países de la Liga Árabe y el Vaticano) y de comunidades que se oponen terminantemente a los derechos LGBT.

34 La única prohibición relativa al derecho al trabajo figura en el apartado a) del párrafo 3 del artículo 8 del PIDCP: “3. a) Nadie será constreñido a ejecutar un trabajo forzoso u obligatorio; b) El inciso precedente no podrá ser interpretado en el sentido de que prohíbe, en los países en los cuales ciertos delitos pueden ser castigados con la pena de prisión acompañada de trabajos forzados, el cumplimiento de una pena de trabajos forzados impuesta por un tribunal competente; c) No se considerarán como 'trabajo forzoso u obligatorio', a los efectos de este párrafo: i) Los trabajos o servicios que, aparte de los mencionados en el inciso b), se exijan normalmente de una persona presa en virtud de una decisión judicial legalmente dictada, o de una persona que habiendo sido presa en virtud de tal decisión se encuentre en libertad condicional; ii) $\mathrm{El}$ servicio de carácter militar y, en los países donde se admite la exención por razones de conciencia, el servicio nacional que deben prestar conforme a la ley quienes se opongan al servicio militar por razones de conciencia. iii) El servicio impuesto en casos de peligro o calamidad que amenace la vida o el bienestar de la comunidad; iv) El trabajo o servicio que forme parte de las obligaciones cívicas normales".

35 Comité de Derechos Humanos de las N.U., Observaciones finales sobre Estados Unidos, 18 de diciembre del 2006, U.N. Doc. CCPR/C/USA/CO/3, \25. 


\section{Conclusión}

A pesar del desarrollo de la protección a las personas LGBT mostrado en los anteriores párrafos, las agencias de Naciones Unidas tales como la OIT y el Alto Comisionado de Derechos Humanos, necesitan poner más atención a estos temas. Específicamente, la OIT debe cambiar su interpretación restrictiva respecto la disposición contra la discriminación por una más incluyente, teniendo en cuenta el importante papel de tal organización en la definición del derecho a un trabajo justo, digno y adecuado. Los pasos que han adelantado los comités de los dos tratados más importantes de derechos humanos en el Sistema Internacional son importantes y deben ser celebrados; sin embargo, el proceso de seguimiento a las recomendaciones dadas a los Estados debe ser más riguroso y se deben aprovechar escenarios multilaterales como el Examen Periódico Universal, para denunciar el no cumplimiento de recomendaciones y comprometer en ellas a los Estados que son persistentes en violaciones de derechos de la comunidad LGBT.

Este texto muestra de qué manera algunas decisiones tomadas en el derecho internacional impactan positivamente la construcción de legislaciones nacionales más incluyentes, como es el caso de Australia y EEUU. Sin embargo, estos esfuerzos son mucho más complejos y arduos si el principal agente internacional que regula y promueve el derecho al trabajo no se compromete con una agenda, interpretación y acciones, incluso impositivas, que garanticen los derechos de las personas LGBT en el empleo. 